\title{
\&BRC
}

\section{Virtual screening of antiviral drugs and natural compounds for potential inhibition of the novel SARS-CoV-2 spike glycoprotein receptor-binding domain}

Shilu Mathew ${ }^{1}$, Fatiha Benslimane ${ }^{1}$, Asmaa A. Althani ${ }^{1,2}$, Hadi M. Yassine ${ }^{1,2 *}$

${ }^{1}$ Biomedical Research Center, Qatar University, Doha, Qatar

2Department of Biomedical Sciences, College of Health Sciences-QU Health, Qatar University, Doha, Qatar

\section{BACKGROUND}

The rapid outbreak of the novel severe acute respiratory syndrome coronavirus 2 (SARSCoV-2) in Wuhan, China that was followed by its wide spread around the world has resulted in the coronavirus disease 2019 (COVID-19) pandemic. To date, it infected over 33 million and resulted in over one million fatalities designating it as a public health emergency of international concern. Although many efforts are being made to contain the pandemic, there lacks a specific therapy to treat COVID-19 and vaccines against SARS-CoV-2 are still under development. To conquer viral infections, inhibiting the spike glycoprotein, which contains the receptor-binding domain (RBD) that mediates the virus's entry to host cells, through antiviral compounds could be an effective therapeutic strategy.

\section{OBJECTIVE}

The aim of this study is to identify novel inhibitors that target the RBD domain in the spike glycoprotein of the SARS-CoV- 2 . Comparative screening of such chemical and natural compounds for SARS-CoV-2 spike inhibitors has not yet been reported.

\section{METHODOLOGY}

- SARS-CoV-2 spike protein was modeled from the recently reported electron microscopy protein structure (PDB ID: 6VSB) and the previously described SARSCoV protein structure (PDB ID: 6ACD and 6ACJ).
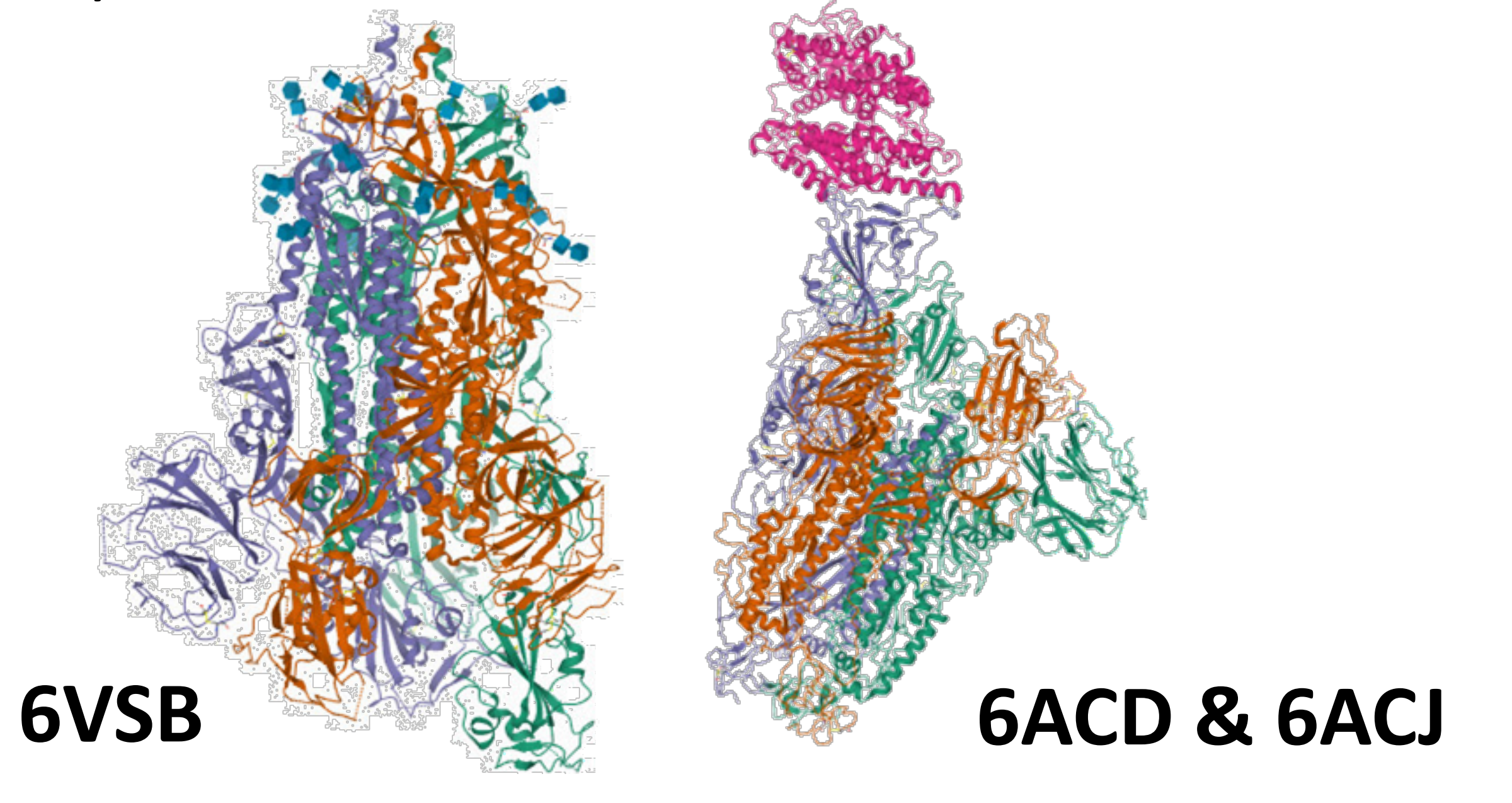

- Virtual lab bench CLC Drug Discovery was used to computationally screen for the inhibitory effects of natural compounds in comparison with current drugs and antiviral drugs in order to predict novel promising therapies against the RBD region of SARSCoV-2 spike protein.

- Quantitative Structure-Activity Relationship (QSAR) were also performed to determine the physicochemical properties and the biological activities of the best binders

\section{References}

S. Arshad Ali, M. Baloch, N. Ahmed, A. Arshad Ali, A. Iqbal, The outbreak of Coronavirus
Disease 2019 (COVID-19)-An emerging global health threat. J Infect Public Health 13, 644-

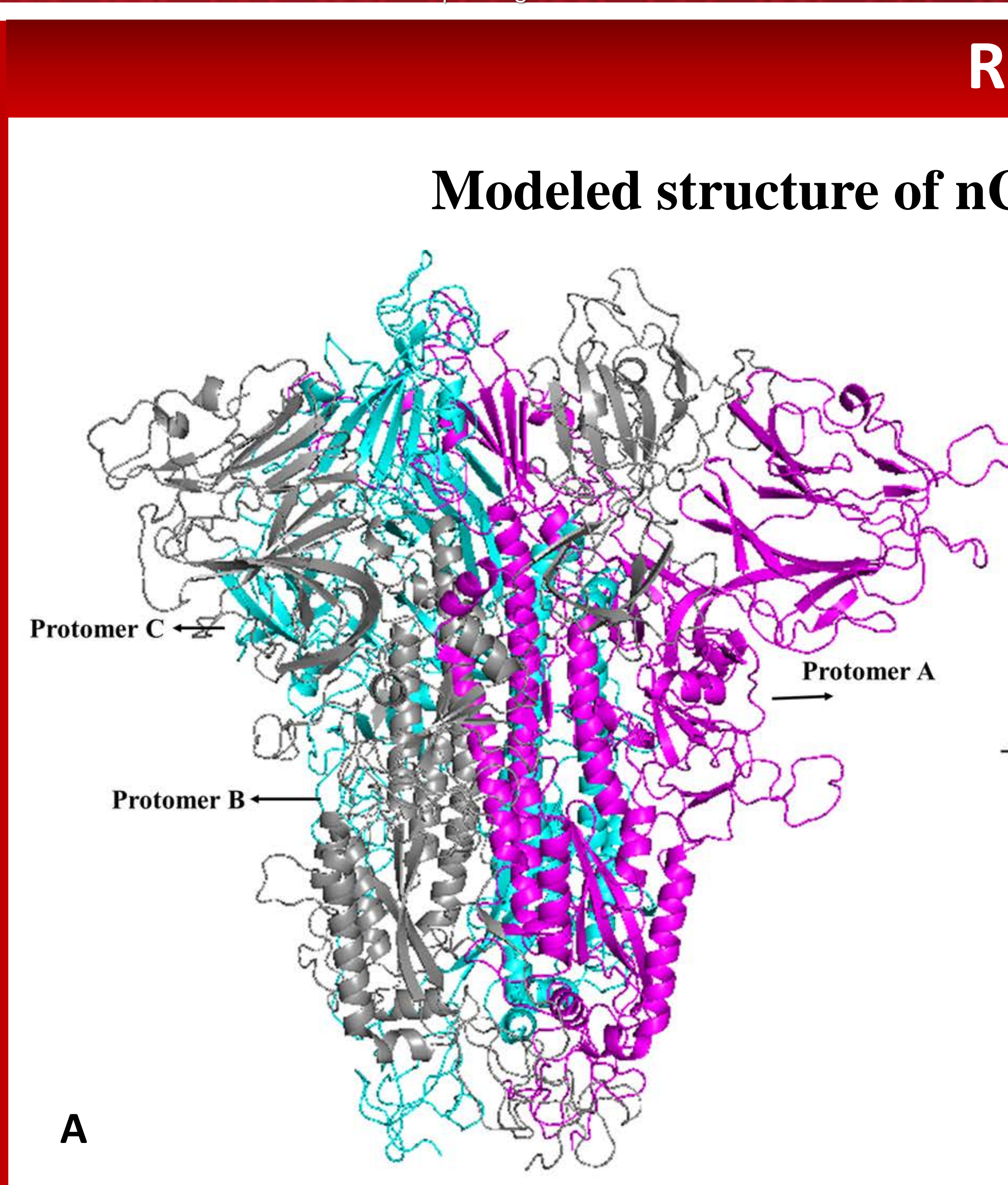

RESULT

Side view

Figure-1: (A) Schematic of modeled nCoV19 Spike trimeric structure colored by protomer (Chain Apink color, Chain B-grey color and Chain C-Cyan color). Side views of the prefusion structure of the nCoV19 Spike protein with a single RBD in the up-conformation. (B) Top view of the prefusion structure of the nCoV19 Spike protein. RBD protomer is shown as surface colored corresponding to the schematic color in A.

\section{Computational Docking studies:}

The below figures illustrates the in-silico docking confirmation of the top five best binders in each tested category to RBD region of $n$ CoV19 spike protein. Modeled nCoV19 spike protein protomer A, $B$ and $C$ are represented as pink, grey and cyan ribbon like structure. The anchored hydrogen bond between the compound and RBD epitopes are shown in as black color.
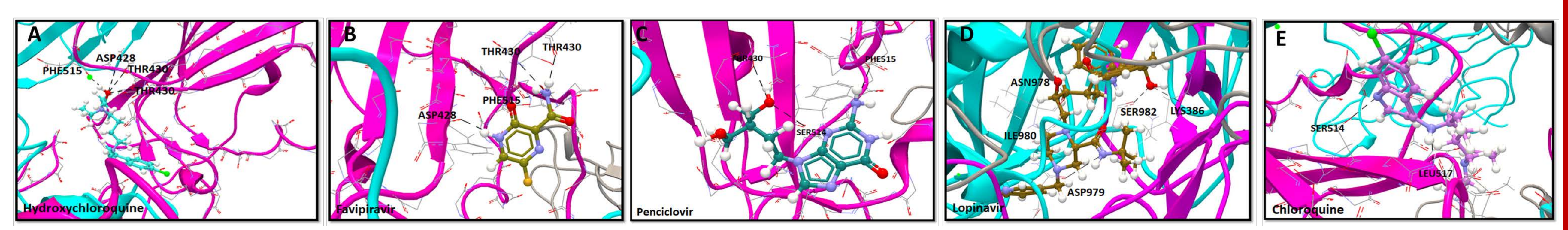

Figure-2: Current COVID-19 treatment drugs
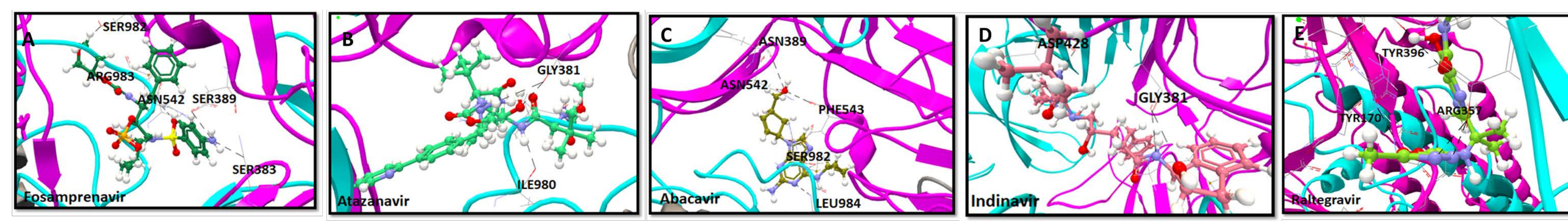

Figure-3: Anti-viral compounds
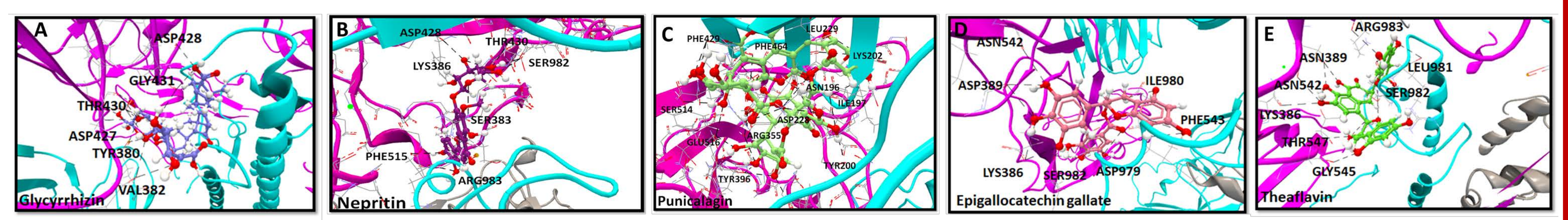

Figure-4: Anti-viral natural compounds
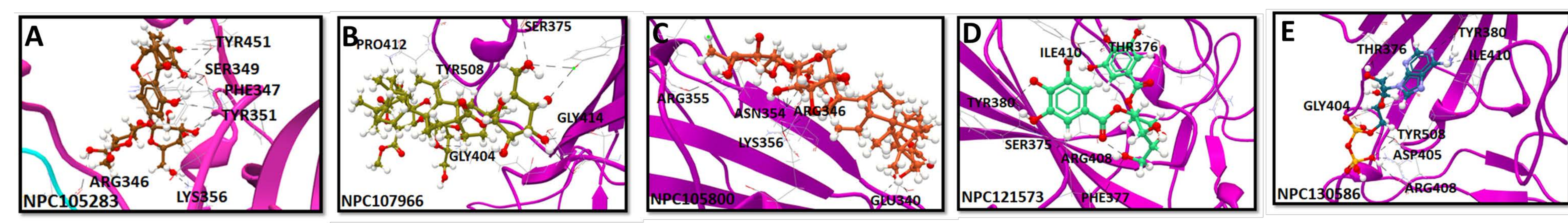

Figure-5: Natural compounds

\section{CONCLUSION}

Using computational screening, we identified potential natural compounds that bind effectively to SARS-CoV-2 spike glycoprotein compared to current COVID-19 treatment drugs. Using structure-based design and combination-based drug therapy, the identified molecules could be used to generate anti- SARS-CoV-2 drug candidates. 